\title{
Xilitol y prevención de otitis media aguda
}

\author{
Xylitol and acute otitis media prevention
}

\author{
Gustavo Bravo C', Noemí Aguirre R², Héctor Bahamonde S $^{1}$.
}

\begin{abstract}
RESUMEN
La otitis media aguda (OMA) es una patología extraordinariamente frecuente en escolares y lactantes, siendo la primera causa de uso de antibióticos en este último grupo. El principal agente involucrado es el S. pneumoniae. Se ha postulado la hipótesis de que el xilitol, un poliol presente en la corteza de abedules, podría tener efectos inhibitorios sobre ésta y otras bacterias otopatógenas en el momento en que se encuentran en la rinofaringe, lo que podría resultar efectivo a la hora de prevenir la invasión de la cavidad timpánica y por ende el desarrollo de OMA. En esta revisión se analizan los estudios realizados que respaldan el uso del xilitol como profilaxis de OMA.
\end{abstract}

\begin{abstract}
Acute otitis media (AOM) is an extremely common disease in school children and infants, which is the first cause of antibiotic use in this latter group. The main agent involved is $\mathrm{S}$ pneumoniae. It has proposed the hypothesis that xylitol, a polyol which is present in the bark of birch, may have inhibitory effects on this bacteria and another otopathogen at the time found in the nasopharynx, which may be effective in preventing the invasion of the tympanic cavity and thus the development of AOM. In this review, is it discussed the studies supporting the use of xylitol prophylaxis of AOM.
\end{abstract}

\section{INTRODUCCIÓN}

El xilitol es un poliol, alcohol con una estructura de cinco átomos de carbono. Presente en la corteza de abedules y también en diversas frutas y verduras ${ }^{1}$. Posee un dulzor similar al de la sucrosa, pero con solamente dos tercios de las calorías. Por este motivo, fue incorporado como suplemento dietético en chicles y pastillas, inicialmente en Europa, pero actualmente se encuentra disponible en gran parte del mundo. La posterior observación de que su uso disminuía la incidencia de caries ${ }^{2-3}$ llevó a que asociaciones odontológicas europeas respaldaran su utilización como profilaxis de esta patología, y fue a partir de ese momento que el xilitol comenzó a ganarse un espacio en la medicina preventiva. Así, surgen nuevos estudios enfocados en buscar un potencial efecto antimicrobiano de este compuesto

\footnotetext{
Médico. Servicio de Otorrinolaringología, Hospital Clínico Universidad de Chile.
}

2 Interna de Medicina, Universidad de Chile. 
sobre otros agentes patógenos presentes en la cavidad oral ${ }^{4}$.

La otitis media aguda (OMA) es una patología extraordinariamente frecuente en lactantes y escolares. Entre los mecanismos involucrados en su génesis existe un ascenso de agentes patógenos desde la rinofaringe a través de la trompa de Eustaquio. En situaciones en que ocurre disfunción tubárica, se modifica el medio en la cavidad timpánica, convirtiéndose en un caldo de cultivo ideal para la proliferación de bacterias y esto lleva al desarrollo de la infección. El principal agente involucrado es el $S$ pneumoniae ${ }^{5}$.

Un grupo finlandés postula la hipótesis de que el xilitol podría tener efectos inhibitorios sobre ésta y otras bacterias otopatógenas en el momento en que se encuentran en la rinofaringe, lo que podría resultar efectivo a la hora de prevenir la invasión de la cavidad timpánica y por ende el desarrollo de OMA. Es así que surge una corriente de investigación que abre nuevas perspectivas para la profilaxis de esta patología ${ }^{6}$. En las siguientes páginas analizaremos los estudios realizados en torno a esta alternativa emergente.

\section{EVIDENCIA MICROBIOLÓGICA}

Comienza en la década de los 70. A partir de un estudio en el que se compararon los efectos de xilitol y sorbitol en caries de ratas ${ }^{7}$, surge la hipótesis de que este poliol podría tener algún efecto sobre la proliferación de $S$ mutans, principal agente causal de caries. Knuutila y cols. observaron en un estudio que xilitol disminuye su crecimiento y afecta su capacidad para acidificar el medio oral ${ }^{8}$.

A partir de esto se desarrolla una línea de investigación que busca aclarar los procesos a través de los cuales actúa el xilitol. Se observa que al exponer a $S$ mutans a esta fuente de carbono, ocurren alteraciones en la composición de la pared celular, disrupción en la síntesis normal de proteínas y alteración de sus capacidades de adhesión a mucosas ${ }^{9-11}$. Esto sería producto de la incorporación del xilitol a las vías metabólicas normales de la bacteria ${ }^{12}$. Este mecanismo es compartido por otros microorganismos de la familia de los estreptococos, entre ellos el pneumococo, por lo que se plantea que su proliferación también se vería afectada al estar expuesto a este compuesto ${ }^{13}$.
Considerando la importancia del pneumococo en la génesis de la otitis media aguda (OMA), se postula que el uso de xilitol podría tener utilidad en la profilaxis de esta patología. Para respaldar esta hipótesis se realizan nuevos estudios. Tapiainen y cols. desarrollaron un protocolo en el que exponen cinco cepas de pneumococo, algunas de ellas otopatógenas demostradas, a seis distintos medios de cultivo, con concentraciones de xilitol al 0,5\% y $5 \%$, glucosa al $5 \%$, fructosa al $5 \%$, sorbitol al $5 \%$ y un medio de control, respectivamente. Se observó que al cabo de dos horas de exposición a xilitol, tanto a concentraciones de $0,5 \%$ como de $5 \%$, aparecen irregularidades morfológicas en la estructura de la pared celular, cambios morfológicos en la cápsula de polisacáridos, disminución significativa de la proliferación en relación a los otros medios de cultivo, aumento significativo en la cantidad de bacterias con daños estructurales y autolisadas, Además se observó una disminución significativa en la capacidad de formar cadenas en relación a los otros medios ${ }^{14}$.

A estas observaciones se suma la evidencia de inhibición significativa en la capacidad de adhesividad de $S$ pneumoniae a la mucosa nasofaríngea al estar expuesto a un medio de cultivo con una concentración de xilitol al 5\%, fenómeno que también fue observado en cepas de $H$ influenzae, otra bacteria con un rol central en la patogenia de OMA ${ }^{15}$.

Todos estos resultados permiten plantear que el uso de xilitol podría ser útil en la profilaxis de OMA. Esto da pie para la realización de los ensayos clínicos que describiremos a continuación.

\section{ENSAYOS CLÍNICOS}

Considerando que la OMA es una patología particularmente frecuente en niños en edad escolar y lactantes, siendo la principal causa de uso de antibióticos en este último grupo ${ }^{16}$, el contar con un medio de profilaxis adquiere una relevancia no menor.

Por lo menos cinco ensayos clínicos han sido realizados para evaluar la efectividad del xilitol como agente profiláctico para esta patología. El primero de estos estudios se publica en 1996: estudio a doble ciego, randomizado, realizado en 11 guarderías de la ciudad de Oulu, Finlandia. Se administra xilitol 
a través de chicles. Participan 306 individuos distribuidos en un grupo control de 149 niños (edad promedio de 4,9 años, DE 1,5 años) que utilizan chicle con sucrosa, y un grupo de 157 niños (edad promedio de 5 años, DE 1,4 años) que utilizan chicle con xilitol. Se utiliza un régimen de administración de dos chicles por cinco veces al día, alcanzando una dosis de $8,4 \mathrm{~g} /$ día. Se realiza un seguimiento de dos meses en los que se monitoriza la ocurrencia de OMA, definida como aparición de síntomas de infección respiratoria alta asociados a signos de efusión del oído medio (membrana timpánica con signos inflamatorios o disminución de la motilidad timpánica objetivada por medio de otoscopía neumática). Se observó una incidencia de OMA de $20,8 \%$ en el grupo control, contra $12,1 \%$ en el grupo asignado a chicles con xilitol diferencia estadísticamente significativa (disminución de 8,7\%; $\mathrm{P}=$ 0,004 con IC $95 \%$ entre $0,4 \%$ y $17 \%$ ). Además se observó una disminución de $10,4 \%$ en la prescripción de antibióticos en el grupo asignado a xilitol, diferencia estadísticamente significativa $(P=0,032$ con IC $95 \%$ entre $0,9 \%$ y $19,9 \%$ ). No se observaron variaciones significativas en la tasa de portación nasofaríngea de $S$ pneumoniae. Se reportaron dos casos de diarrea en niños en el grupo asignado a xilitol, lo que podría constituir un efecto adverso asociado a este compuesto ${ }^{17}$.

El diseño de este estudio no permite un uso adecuado del compuesto en lactantes debido a la incapacidad para mascar chicle, lo que es particularmente relevante debido a que el pico de incidencia de OMA ocurre entre los 6 y 18 meses $^{18}$. Tomando estos datos en consideración, se realiza un segundo estudio, en 1998: se reclutan 857 niños sanos. En función de la capacidad de mascar chicle se dividió a los niños en un grupo de 324 individuos asignados a xilitol en dosis de $10 \mathrm{~g}$ por día vía jarabe (165 niños) 0 administración de jarabe con xilitol en dosis de $0,5 \mathrm{~g}$ por día a modo de control (159 niños); y un grupo de 533 individuos asignados a xilitol en dosis de $8,4 \mathrm{~g}$ por día vía chicle (179 niños), administración de xilitol en dosis de $10 \mathrm{~g}$ por día vía pastillas (176 niños) o administración de xilitol en dosis de 0,5 g por día vía chicle a modo de control (178 niños). Se realizó un seguimiento de 3 meses, monitorizando la ocurrencia de OMA. Se observó al menos un evento de OMA en el $41 \%$ de los niños asignados a jarabe control, contra $29 \%$ en el grupo de niños asignados a jarabe con xilitol, diferencia estadísticamente significativa ( $P=0,028$ con IC $95 \%$ entre $4,6 \%$ y $55,4 \%$ ). En el grupo asignado a chicle control se observó al menos un evento de OMA en el $28 \%$ de los niños, contra $16 \%$ en el grupo asignado a chicle con xilitol, diferencia estadísticamente significativa ( $P=0,025$ con IC $95 \%$ entre $10 \%$ y $71,1 \%$ ). No hubo diferencias significativas entre el grupo asignado a pastillas con xilitol y el grupo control. Hubo también disminuciones significativas en la prescripción de antimicrobianos en los grupos asignados a jarabe con xilitol a dosis de $10 \mathrm{~g}$ por día y chicle con xilitol a dosis de 8,4 g por día ${ }^{19}$. Nuevamente se observa que el uso de xilitol es efectivo como profilaxis de OMA, esta vez agregando la posibilidad de usarlo en forma de jarabe en niños incapaces de usar chicle. Considerando los buenos resultados obtenidos en ambos estudios, se realiza un tercer ensayo clínico enfocado en el uso de xilitol para el manejo de pacientes con historia de OMA recurrente 0 efusión crónica del oído medio. Para esto se reclutan 270 niños portadores de tubos de timpanostomía. Se dividen en tres grupos: a 90 niños se les administró xilitol y se dio instrucciones en medidas de higiene a sus padres, al resto se les asignó a dos grupos, uno solamente con instrucciones en medidas de higiene, el otro se utilizó como control. No se observaron diferencias significativas entre los grupos ${ }^{20}$.

Buscando una alternativa más práctica de administración, se diseñó un cuarto estudio con un régimen de xilitol dividido en tres tomas diarias, esquema que se aplicó a grupos asignados a xilitol en chicles 0 en jarabe, comparado con grupos de control. Las dosis totales diarias fueron similares a las de ensayos anteriores. Se reclutaron 663 niños sanos y se realizó seguimiento de tres meses. No se observaron diferencias significativas en la ocurrencia de OMA entre los distintos grupos ${ }^{21}$.

En un nuevo intento de encontrar esquemas más simples, se realiza un quinto ensayo clínico en el que se intenta la administración de xilitol en cinco tomas diarias, por vía de jarabe, chicle o pastillas, pero solamente durante episodios de infecciones respiratorias altas. Se reclutan 1277 niños que son randomizados en grupos siguiendo los mismos principios aplicados a estudios anteriores, con dos grupos de control, y se realiza un seguimiento de 4 meses. No se observan diferencias estadísticamen- 
te significativas en la incidencia de OMA entre los grupos, lo que se interpreta como fracaso de este esquema ${ }^{22}$.

Respecto a la forma de hacer profilaxis, surgen estudios que proponen iniciar la exposición a xilitol en mujeres embarazadas, como una forma de disminuir la trasmisión materna de estos patógenos. Se realizó un ensayo clínico en 2010 en Japón, que seleccionó a 107 mujeres embarazadas con altos niveles salivales $S$ Mutans, ramdomizadas en un grupo que recibe chicle con xilitol $(n=56)$ y otro grupo control que solo recibe medidas de higiene habituales $(n=51)$. Las participantes reciben xilitol en chicle ( $1,32 \mathrm{~g}$ de xilitol) por lo menos 4 veces al día iniciando en el sexto mes de embarazo y terminó 13 meses después. Los niños nacidos del grupo con xilitol mostraron una menor colonización de $S$ Mutans respecto al grupo de control en forma significativa (a los 12 mes es 10,2\% v/s 45,5\% de coIonización $p<0,001)$. Además los niños nacidos del grupo control adquirieron $S$ Mutans 8,8 meses antes que los del grupo con xilitol $(p<0,001)$, lo cual permitiría una estrategia importante de prevención de caries para los niños, dejando la puerta abierta para estudiar los efectos para $0 \mathrm{MA}^{23}$.

Finalmente, el año 2010 se llevó a cabo una revisión sistemática para evaluar la evidencia sobre Xilitol como profilaxis para OMA en niños. Se utilizó PubMed y otras bases para identificar la evidencia. Se revisó 1.479 títulos y se excluyeron 1.435. Se revisaron los 44 restantes, solo 4 ensayos clínicos aleatorios controlados cumplieron los criterios de inclusión, los cuales se incluyen en nuestra exposición de ensayos clínicos mencionada previamente ${ }^{24}$.

Con esta evidencia se sugiere que en esquemas de administración continua de cinco tomas diarias el xilitol a dosis de $10 \mathrm{~g} /$ día sería efectivo para la profilaxis de OMA, utilizado en forma de jarabe como en forma de chicle, siendo bien tolerado, con pocos efectos colaterales ${ }^{24}$. Sin embargo, esquemas de administración más simples no tendrían la misma eficacia. A esto se suman varias otras dudas, las que serán analizadas a continuación.

\section{DISCUSIÓN}

La situación actual con respecto a la utilización del xilitol en clínica no es clara. Aunque los resulta- dos en los ensayos clínicos parecen alentadores, existen diversas aéreas que deben ser discutidas.

Analizando la evidencia expuesta anteriormente, parecen existir suficientes fundamentos microbiológicos para sostener que el xilitol podría ser efectivo en la profilaxis de OMA. No podemos cuestionar lo beneficioso que resulta contar con un medio de prevención para esta patología: considerando su importancia epidemiológica y el uso de antimicrobianos asociado, disminuir su incidencia implica una disminución en las posibles secuelas en los individuos afectados y de los efectos adversos asociados al uso de antibióticos ${ }^{25}$, disminución de la resistencia de microorganismos ${ }^{26}$, y en suma una disminución en los costos de salud ${ }^{27}$.

Sin embargo, debemos considerar que existen argumentos que se oponen a aceptar de forma inmediata esta nueva alternativa de profilaxis. En primer lugar, debemos considerar que un esquema de administración de cinco veces por día es poco práctico para uso clínico debido a que no asegura una buena adherencia. Un estudio sugiere que alcanzar altos picos de concentración de xilitol en saliva sería más importante que el tiempo durante el que se logra mantener su concentración en los rangos en que se observa el efecto antimicrobiano de este compuesto, de lo que se infiere que una administración a frecuencias menores podría ser efectiva para lograr profilaxis ${ }^{28}$. Aunque hemos visto resultados de un régimen de administración de tres veces por día que no fueron favorables, se pueden plantear nuevas alternativas.

En segundo lugar, aún no existe absoluta claridad respecto a los mecanismos de acción del xilitol. Aunque la evidencia microbiológica ofrece algunas respuestas, no aclara todas las dudas, considerando la observación de que al cabo de dos meses de utilización de xilitol no existen diferencias significativas en la portación nasofaríngea de pneumococo ${ }^{17}$. Un tercer aspecto a considerar son los efectos adversos que podrían asociarse a la utilización de xilitol. Se sabe que es absorbido en bajas cantidades por el intestino, esto explica que su uso en dosis altas pueda provocar diarrea. Se realizó un estudio para establecer la tolerancia a xilitol administrado por vía oral a 60 niños, con un seguimiento de tres meses. Se administraron dosis de $5 \mathrm{~g}$ y de $7,5 \mathrm{~g}$ en esquemas de tres veces por día y una vez por día, respectivamente. Comparados con grupos a los que se les 
administró placebo, no se observó una diferencia significativa en la incidencia de diarrea en los individuos que ingirieron xilitol ${ }^{30}$. Sin embargo, existe un estudio que sugiere que dosis de $8,4 \mathrm{~g}$ sí se puede relacionar con problemas gastrointestinales en lactantes ${ }^{31}$. Estas observaciones contradictorias obligan a realizar más estudios, considerando que toda esta evidencia está limitada a periodos de tiempo no mayores a tres meses.

Una cuarta arista corresponde a las características raciales de los individuos en que se han realizado estos estudios. Los principales ensayos clínicos se realizaron en Finlandia. Es imposible asegurar que los resultados se verán homologados en individuos de otras etnias ${ }^{32}$.

Un quinto aspecto a considerar es la forma de administración. La administración en chicles puede ser un peligro de asfixia en niños pequeños, muchas escuelas los prohíben, lo cual sería un obstáculo en programas de prevención ${ }^{24}$. Al respecto se conoce una nueva forma de administración vía spray nasal, que pareciera ser una buena alternativa, pero se requiere de estudios.

Por último, se debe analizar los costos que implica utilizar esta profilaxis a gran escala. No existe disponibilidad de chicles con contenido de xilitol en todos los mercados, menos aún de jarabe con contenido de xilitol, lo que implica que como primera etapa para una intervención se requiere introducir este producto en muchas regiones. Además hay que considerar que se ha estimado que se necesitaría 5.600 chicles con xilitol para prevenir un único episodio de OMA utilizando los esquemas de dosificación propuestos en los ensayos clínicos que han tenido resultados positivos ${ }^{28}$ (régimen de dos chicles por cinco veces al día). Estos resultados no resultan alentadores desde una perspectiva estrictamente económica.

\section{CONCLUSIÓN Y RECOMENDACIONES FINALES}

Al analizar la evidencia existente, no cabe duda de que el xilitol podría llegar a convertirse en una alternativa viable para la profilaxis de OMA. Sin embargo, no debemos dejarnos llevar por esta emocionante perspectiva: aún quedan muchas preguntas por responder y mucho camino por avanzar. Los argumentos en contra de la utilización del xilitol en forma masiva en este momento son varios, y en particular deben ser considerados cuando pensamos que el uso de este compuesto implica una intervención a gran escala en niños de corta edad, particularmente vulnerables y con toda una vida por delante. Sin mayor información sobre efectos adversos, poca claridad sobre los costos económicos, y falta de esquemas cómodos de utilización, es difícil plantear que este camino sea el más conveniente en este momento. Sin embargo, no podemos descartar que llegue a serlo en el futuro. Por esta razón, creemos que lo más adecuado es ser paciente y observar cómo avanza esta línea de investigación que nos permita el día de mañana tomar una postura definitiva con respecto al uso de xilitol en la profilaxis de la otitis media aguda.

\section{BIBLIOGRAFÍA}

1. MäkInEN K, SöderLlingE. A quantitative study of mannitol, sorbitol, xylitol and xylose in wild berries and commercial fruits. J Food Science 1980; 45: 367-71.

2. Mäkinen K, Bennett $C$, Hujoel P et al. Xylitol chewing gums and caries rates: a 40-month cohort study. J Dent Res 1995; 74: 1904-13.

3. Burton L. Edelstein. Xylitol syrup can reduce dental caries progression in young children. $J$ of Pediatrics 2010; 156-60.

4. Kontiokari T, Uhari M, Koskela M. Effect of xylitol on growth of nasopharyngeal bacteria in vitro. Antimicr Agents Chemot 1995; 39: 1820-3.

5. Bluestone $C$, Klein J. Otitis media in infants and children. Philadelphia, PA: W.B. Saunders, 1995.

6. Kontiokari T, Uhari M, Koskela M. Antiadhesive effects of xylitol on otopathogenic bacteria. $J$ Antimicr Chemoth 1998; 41: 563-656.

7. Muehlemann H, Regolati B, Marthaler T. The effect on rat fissure caries of xylitol and sorbitol. Helvetica Odontologica Acta 1970; 14: 48-50.

8. KnuUttila M, Makinen K. Effect of xylitol on the growth and metabolism of Streptococcus mutans. Caries Research 1975; 9: 177-89.

9. Tuompo H, Meurman J, Lounatmaa K et al. Effect of xylitol and other carbon sources on the cell wall of Streptococcus mutans. Scan J Dent Res 1983; 91: 17-25.

10. Hrimech M, Mayrand D, Grenier D et al. Xylitol disturbs protein synthesis, including the 
expression of HSP-70 and HSP-60, in Streptococcus mutans. Oral Microbiology and Immunology 2000; 15: 249-57.

11. Söderling E, Alaräisänen L, Scheinin A et al. Effect of xylitol and sorbitol on polysaccharide production by and adhesive properties of Streptococcus mutans. Caries Research 1987; 21: 109-16.

12. Trahan L, Bareil M, Gauthier L et al. Transport and phosphorylation of xylitol by a fructose phosphotransferase system in Streptococcus mutans. Caries Research 1985; 19: 53-63.

13. Assev S, Waler $S$ \& Rölla G. Further studies on the growth inhibition of some oral bacteria by xylitol. Act Pat Imm Scandinavica 1983; 91: 261-5.

14. TAPIAINEN T, SORMUnen $R$ y cols. Ultrastructure of Streptococcus pneumoniae after exposure to xylitol. J Antimicr Chemotherapy 2004; 54: 225-8.

15. Kontiokari T, Uhari M, Koskela M. Antiadhesive effects of xylitol on otopathogenic bacteria. $J$ Antimicr Chemotherapy 1998; 41: 563-5.

16. Finkelstein JA, Metlay JP, Davis Rl y cols. Antimicrobial use in defined populations of infants and young children. Arch Pediatric \& Adolescent Medicine 2000; 154: 395-400.

17. Uhari M, Kontiokari T, Koskela M. \& Niemelä M. Xylitol chewing gum in prevention of acute otitis media: double blind randomised trial. BMJ1996; 313: 1180-4.

18. Reval K, Dobbs L y cols. Incidence of Acute Otitis Media and Sinusitis Complicating Upper Respiratory Tract Infection: The Effect of Age. Pediatrics 2007; 119: 1408-12.

19. Uhari M, Kontiokari T \& Niemelä M. A novel use of xylitol sugar in preventing acute otitis media. Pediatrics 1998; 102: 879-84.

20. Uhari M, TapiaInen T. \& KontIOKaRI T. Xylitol in preventing acute otitis media. Vaccine 2001; 19 : S144-S147.

21. Hautalahti 0 , Renko M, Tapiainen T y cols. Failure of Xylitol Given Three Times a Day for Preventing Acute Otitis Media. Pediatrics Infectious Disease Journal 2007; 26: 423-7.
22. Tapiainen T, Luotonen L, Kontiokari T, y cols. Xylitol administered only during respiratory infections failed to prevent acute otitis media. Pediatrics 2002; 109: e19.

23. Nakai Y, Shinga-Ishihara C, KaJi M, Moriya K, Murakami-Yamanaka K and Takimura M. Xylitol Gum and Maternal Transmission of Mutans Streptococci. J Dent Res 2010; 89(1): 56-60.

24. Danhauer L, Johnson E, Corbin E, Bruccheri G. Xylitol as a prophylaxis for acute otitis media: Systematic review. Inter J Audio 2010; 49: 75476.

25. DANCER S. How antibiotics can make us sick: the less obvious adverse effects of antimicrobial chemotherapy. Lancet Infect Diseases 2004; 4: 611-9.

26. Seppa" la" H, Klaukka T, Vuopio-Varkila J, et al. The effect of changes in the consumption of macrolide antibiotics on erythromycin resistance in group A streptococci in Finland. N E J Med 1997; 337: 441-6.

27. McGowan JE JR. Economic impact of antimicrobial resistance. Emerging Infectious Diseases 2001; 7: 765.

28. Tapiainen T, Renko M, Kontiokari T \& Uhari M. Xylitol Concentrations in the Saliva of Children After Chewing Xylitol Gum or Consuming a Xylitol Mixture. Eur J Microb Infectious Diseases 2002; 21: 53-5.

29. Miтchell AA. Xylitol prophylaxis for acute otitis media: tout de suite? Pediatrics 1998; 102: 974-5.

30. Vernacchio L, Vezina RM \& Mitchell AA. Tolerability of oral xylitol solution in young children: Implications for otitis media prophylaxis. International Journal of Pediatric Otorhinolaryngology 2007; 71: 89-94.

31. Akerblom HK, Koivukangas KT, Puuka R \& Mononen $M$. The tolerance of increasing amounts of dietary xylitol in children. International Journal for Vitamin and Nutrition Research 1982; 22: 53-66.

32. Wright PF. Xylitol sugar and acute otitis media. Pediatrics 1998; 102: 971-2. 